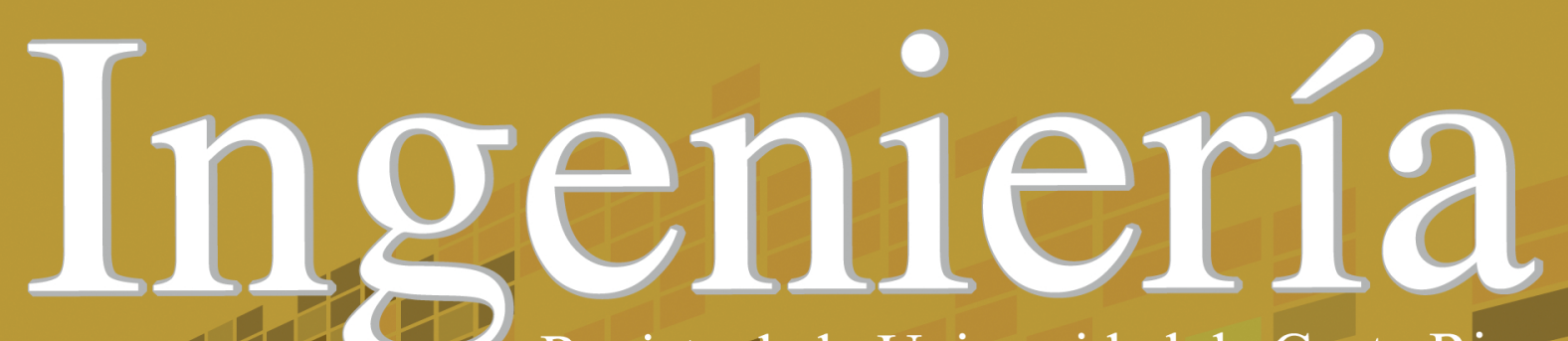

Revista de la Universidad de Costa Riea ENERO/JULIO 2013 - VOLUMEN 23 Número (1)

H

H

H71

Tr.

$x^{2}$

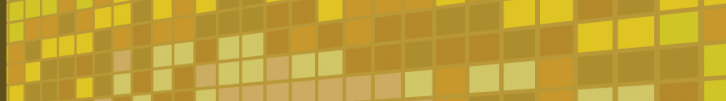

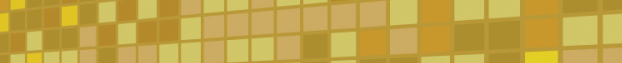

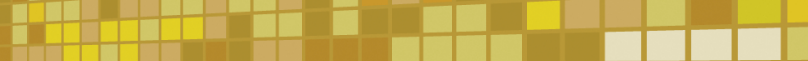

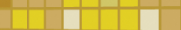

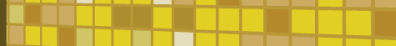

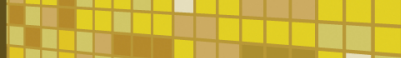

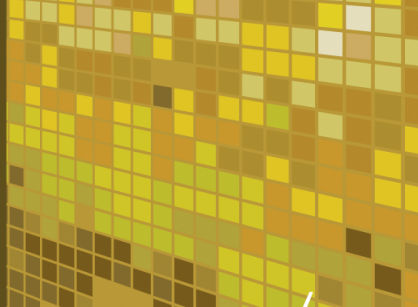

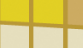



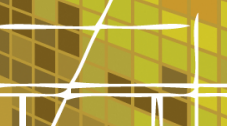

$+\infty \square$ 
Notas técnicas 



\title{
ESTIMACIÓN DE LA INCERTIDUMBRE EN MEDICIONES QUÍMICAS: UN EJEMPLO PRÁCTICO Y SIMPLE
}

Bryan Calderón Jiménez

\begin{abstract}
Resumen
La estimación de la incertidumbre en las mediciones químicas es realizada de manera formal, según los lineamientos descritos en la Guía ISO para la Expresión de la Incertidumbre de Mediciones (GUM), sin embargo, el método de Kragten es una aproximación numérica que puede emplearse para estimar la incertidumbre de medición. El siguiente documento desarrolla la estimación de la incertidumbre de un mensurando de naturaleza química empleando el método de Kragten. Los resultados obtenidos por este método numérico son comparados con los obtenidos por el método clásico propuesto por GUM. Con lo anterior, se pretende otorgar a los laboratorios de ensayos un ejemplo práctico y simple para la estimación de la incertidumbre en mediciones químicas.
\end{abstract}

Palabras clave: Kragten, GUM, incertidumbres, método numérico, mediciones químicas.

\begin{abstract}
The estimation of measurement uncertainty in chemistry is formally made, along the lines described in the ISO Guide to the Expression of Uncertainty in Measurement (GUM), however, the Kragten method is a numerical approximation that can be used to estimating the measurement uncertainty. The following paper develops the estimation of the uncertainty of a chemical measurand using the Kragten method. The results obtained by this method are compared with those obtained by the classical method proposed by GUM. This is intended to provide to the chemical laboratories simple and practical example for estimating uncertainty in chemical measurements.
\end{abstract}

Keywords: Kragten, GUM, uncertainty, numerical method, chemical measurement.

\section{INTRODUCCIÓN}

El término incertidumbre de medida es definido en el Vocabulario Internacional de Metrología de la siguiente manera: "Parámetro asociado al resultado de una medición, que caracteriza la dispersión de los valores que podrían ser razonablemente atribuidos a un mensurando" (ISO, 2007). Una manera sencilla de comprender el concepto de incertidumbre de medida es observarlo como la indicación cuantitativa de la calidad de nuestros resultados. Sin tal indicación no podríamos comparar los resultados, con otros valores de referencia emitidos en especificaciones o normas. Por tanto, es preciso para el quehacer científico evaluar, estimar y expresar la incertidumbre de medida de una manera idónea. Actualmente, la Organización Internacional de Normalización (ISO por sus siglas en ingles) en su "Guía para la Expresión de la Incertidumbre de Medida" (GUM), establece los lineamientos generales para la evaluación y expresión de la incertidumbre de medida en un amplio espectro de mediciones (ISO, 1995). El uso de esta Guía es avalado por la Oficina Internacional de Pesas y Medidas, BIPM por sus siglas en francés. Sin embargo, alternativamente como herramienta 
de cálculo, existe un método numérico rápido y universal que es aplicable para la estimación de incertidumbre empleando hojas de cálculo en MS Excel®, este método es conocido como aproximación numérica ó método de Kragten (Kragten, 1994). El método se fundamenta, al igual que la GUM, en las fórmulas generales de la ley de propagación de la incertidumbre, calculando las desviaciones típicas sin violar la condición de independencia mutua. A continuación se desarrollará como ejemplo práctico la estimación de la incertidumbre para el método AOAC 930.29, el cual consiste en la determinación del contenido de proteína en leche en polvo (AOAC, 1990). Este ejercicio pretende entregar al lector un ejemplo práctico y simple para la estimación de incertidumbre de medición, el cual podrá ser aplicado y adaptado a una gran cantidad de determinaciones en química analítica.

\section{PROCESO DE ESTIMACIÓN DE LA INCERTIDUMBRE DE MEDICIÓN}

La estimación de la incertidumbre es en principio un proceso simple. Los pasos involucrados en el proceso de estimación y evaluación de la incertidumbre en una medición química se pueden resumir de la siguiente manera (Ellison y Williams, 2010):

(1) Establecimiento del mensurando $\rightarrow$

(2) Identificación de las fuentes de incertidumbre

(3) Cuantificación de los componentes de incertidumbre $\rightarrow$

(4) Cálculo de la incertidumbre combinada e incertidumbre expandida.

A continuación desarrollaremos los pasos descritos anteriormente para la estimación de la incertidumbre de la determinación del contenido de proteína en leche en polvo descrita en el AOAC 930.29.

\section{ESTABLECIMIENTO DEL MENSURANDO}

El mensurando en química analítica usualmente se expresa en términos de la concentración de analito que se desea cuantificar. Por lo tanto, es importante tener claridad de lo que se esta midiendo, en otras palabras, se debe estudiar con detalle cada magnitud o cantidad de entrada que define al mensurando, incluyendo correcciones por efectos sistemáticos previamente conocidos. Para poder tener certeza en la estimación de la incertidumbre, es conveniente realizar antes de la definición del mensurando, una breve descripción del proceso de medición con la finalidad de entender a cabalidad el procedimiento de análisis e identificar las posibles fuentes de incertidumbre asociadas al mensurando. Este procedimiento puede venir acompañado de un diagrama de flujo, el cual entregará al analista una visión global del proceso. Para el caso específico de la determinación del contenido de proteína en leche en polvo se podría plantear el siguiente resumen del procedimiento de medición.

\subsection{Procedimiento de medición}

Se pesa $1,00 \mathrm{~g}$ de muestra en un matraz de digestión de Kjeldahl. Se le adiciona a la muestra un catalítico compuesto por $\mathrm{CuSO}_{4}, \mathrm{~K}_{2} \mathrm{SO}_{4}$ y $\mathrm{H}_{2} \mathrm{SO}_{4}$. Se digiere la muestra calentándola suavemente hasta que deje de producir espuma, luego se calienta enérgicamente hasta que se genere una disolución de coloración verde claro. Una vez digerida la muestra, se destila por medio de arrastre de vapor de agua. Los vapores y el condensado provenientes del sistema de destilación son recolectados en un erlenmeyer que contiene una disolución de ácido bórico. Se valora la disolución contenida en el erlenmeyer con $\mathrm{HCl} 0,10 \mathrm{~mol} \mathrm{~L}^{-1}$.

La mayoría de los resultados en las determinaciones analíticas provienen de otras cantidades o magnitudes como por ejemplo: el volumen, la masa, la concentración, el área de los picos de un cromatograma, entre otros. Estas magnitudes de entrada se combinan en forma de una ecuación, la cual puede traer intrínseca operaciones matemáticas básicas (e.j adición, sustracción, multiplicación). Esta ecuación es llamada modelo de medición, la cual es el mensurando (ISO, 1995) y se expresa matemáticamente de acuerdo con la siguiente función de medición: 


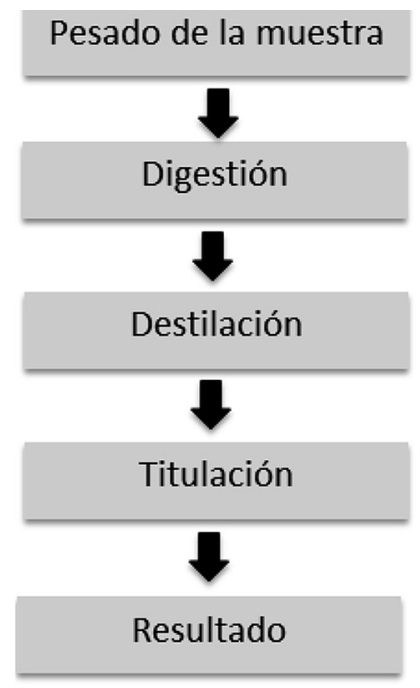

Figura 1. Diagrama de flujo de la determinación del contenido de proteína en lecha en polvo.

Fuente: Elaboración propia, 2012.

$Y=f\left(X_{1}, X_{2}, \ldots, X_{N}\right)$

Donde $X_{1}, X_{2}, \ldots, X_{N}$ son simplemente las magnitudes o cantidades de entrada, $Y$ es el resultado de la medición, $f($ ) denota la ecuación o relación funcional de estas variables. En la práctica lo común es realizar una estimación de entradas $x_{1}, x_{2}, \ldots, x_{N}$ de los valores de $\mathrm{N}$ magnitudes $\mathrm{X}_{1}, \mathrm{X}_{2}, \ldots, \mathrm{X}_{\mathrm{N}}$, para así obtener una estimación del mensurando, representada como y. La expresión matemática que ejemplifica lo anterior viene dada por:

$y=f\left(x_{1}, x_{2}, \ldots, x_{N}\right)$

Para el caso específico de nuestro ejemplo, podríamos definir el mensurando de la siguiente manera.

\subsection{Mensurando para la determinación del contenido de proteína en leche en polvo}

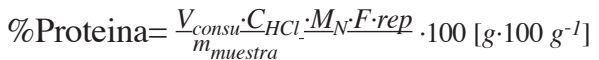

Donde:

\% Proteína: Contenido de proteína en leche en polvo [g $100 \mathrm{~g}^{-1}$ ]

$V_{\text {consu }}$ : Volumen consumido de la disolución de $\mathrm{HCl}\left(\mathrm{V}_{\text {punto final }}-\mathrm{V}_{\text {blanco }}\right)[\mathrm{L}]$

$C_{H C l}$ : Concentración de la disolución de $\mathrm{HCl}$ [mol L-1]

$M_{N}$ : Masa molar del nitrógeno [ $\left.\mathrm{g} \mathrm{mol}^{-1}\right]$

$m_{\text {muestra }}$ : Masa de muestra de leche [g]

rep: Factor adimensional de repetibilidad

$F$ : Factor de conversión de nitrógeno a proteína (6,38 para leche en polvo)

\section{IDENTIFICACIÓN DE FUENTES DE INCERTIDUMBRE}

En términos prácticos para poder definir bien el mensurando es necesario realizar un listado de las magnitudes de influencia y fuentes de incertidumbre relevantes al método analítico de medición. Este listado de magnitudes de influencia se pueden representar gráficamente, esta representación procurará identificar las principales fuentes de incertidumbre $\mathrm{y}$ disminuye el riesgo de omitir o discriminar 
fuentes de incertidumbre que sean importantes para la estimación de la incertidumbre (Ellison, y Barwick, 1998). Para tal efecto es recomendable realizar un diagrama de causa y efecto (también conocidos como diagramas de Ishikawa ó espina de pescado). Las Figuras 2, 3 y 4, representan los posibles diagramas de causa y efecto para la determinación del contenido de proteína en leche en polvo.

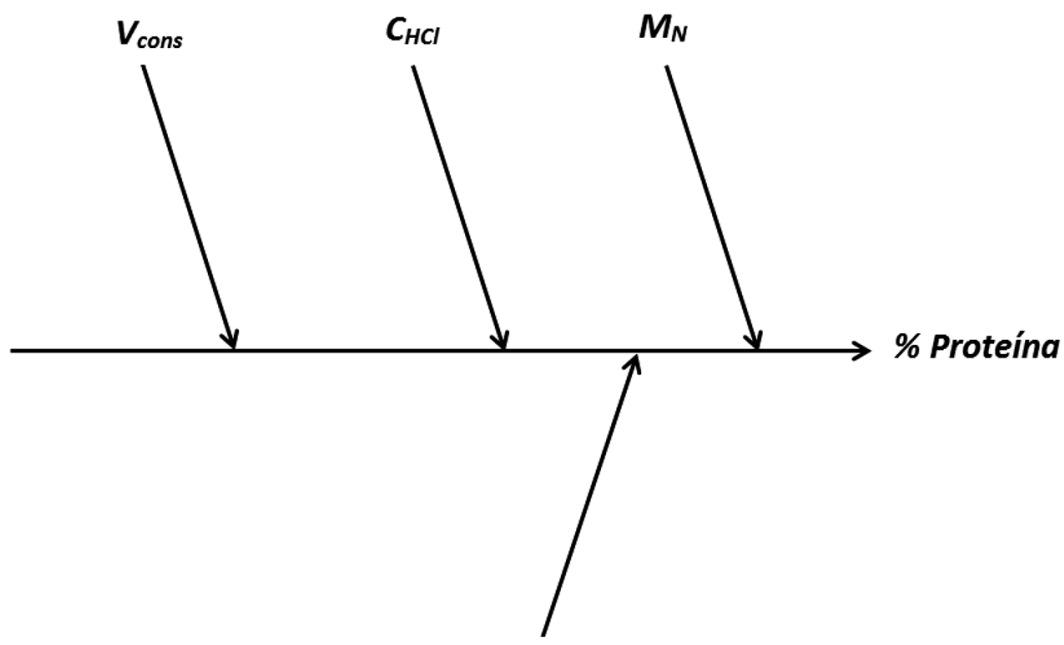

$\boldsymbol{m}_{\text {muestra }}$

Figura 2. Diagrama simple de causa y efecto para la determinación del contenido de proteína en leche. En este diagrama se detallan únicamente las magnitudes de influencia.

Fuente: Elaboración propia, 2012.

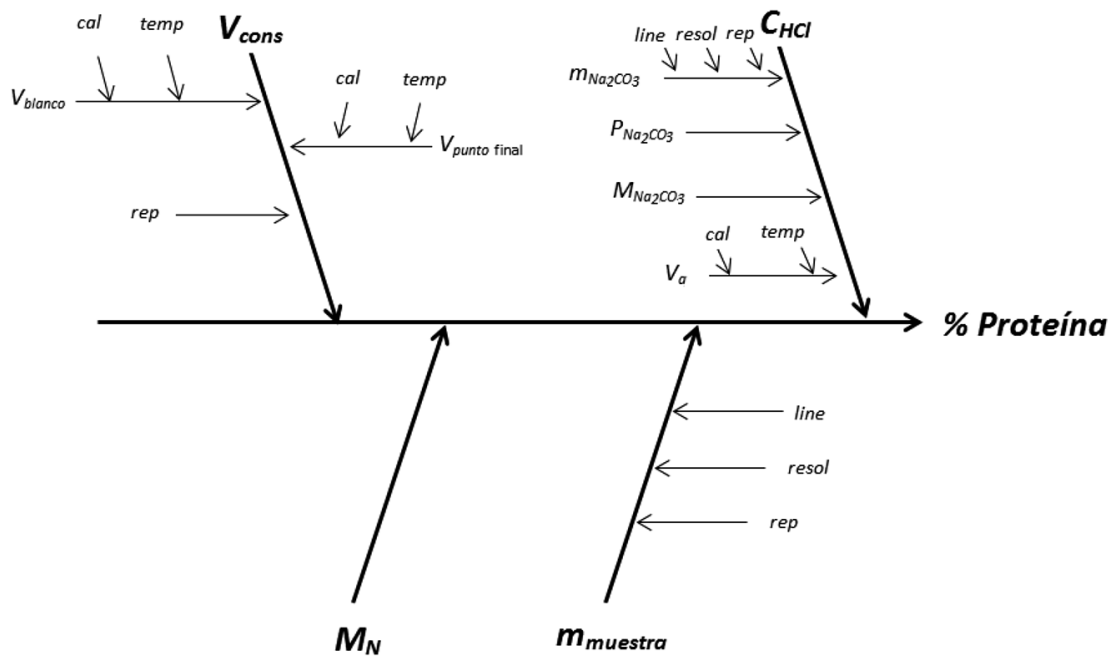

Figura 3. Diagrama detallado de causa y efecto para la determinación del contenido de proteína en leche. En este diagrama se contemplan las posibles fuentes de incertidumbre como por ejemplo la repetibilidad (rep), calibración del equipo de medición (cal), efecto de la temperatura (tem) y resolución (resol) de los equipos de medición.

Fuente: Elaboración propia, 2012. 


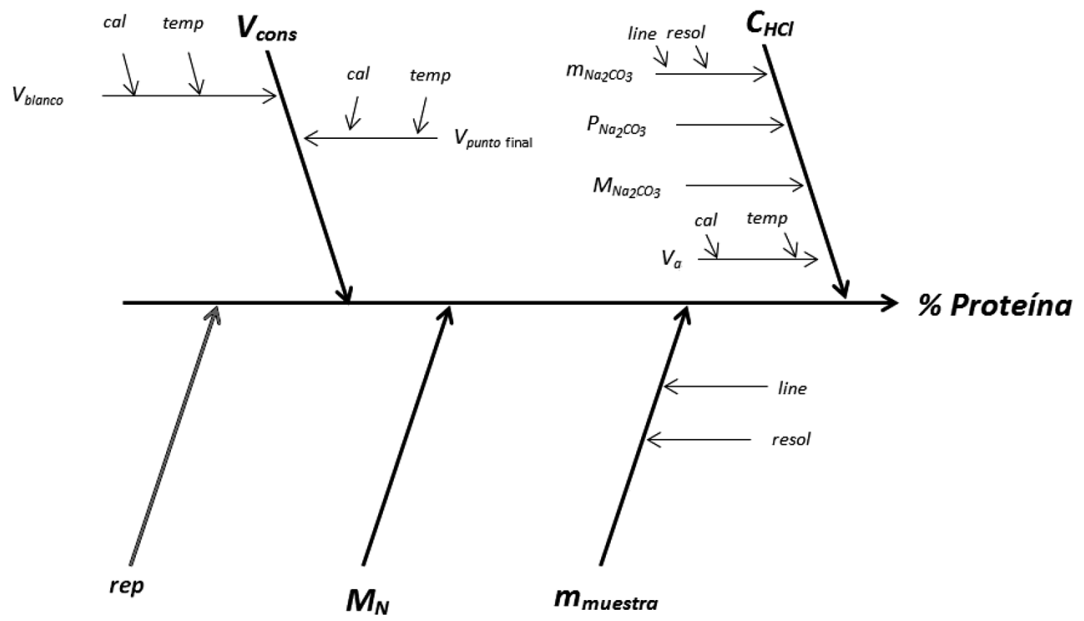

Figura 4. Diagrama detallado de causa y efecto para la determinación del contenido de proteína en leche. En este diagrama las fuentes de incertidumbre por repetibilidad (para este ejemplo la repetibilidad de las determinaciones de masa proviene de la desviación típica de medir sucesivamente una masa patrón o masa control) y se incluyen al modelo del mensurando como una magnitud de influencia como se puede apreciar en la Ecuación (3).

Fuente: Elaboración propia, 2012.

\section{CUANTIFICACIÓN DE LOS COMPONENTES DE INCERTIDUMBRE}

Una vez identificadas las fuentes de incertidumbre asociadas a la medición, se procede con la estimación de la incertidumbre típica para cada una de las magnitudes de influencia.

\subsection{Volumen consumido}

El volumen consumido de $\mathrm{HCl} 0,10 \mathrm{~mol} \mathrm{~L}^{-1}$ se obtiene al sustraer el volumen consumido en la valoración del blanco reactivo $\left(V_{\text {blanco }}\right)$ al volumen consumido en el punto final $\left(V_{\text {punto final }}\right)$ de la valoración de los iones $\mathrm{H}_{2} \mathrm{BO}_{3}{ }^{-}$, en ambos casos como se puede apreciar en la Figura 3 existen dos fuentes o contribuciones de incertidumbre:

\section{Calibración de la bureta}

Los fabricantes de buretas establecen que la precisión (tolerancia) para una bureta de $50 \mathrm{~mL}$ de volumen es de $\pm 0,05 \mathrm{~mL}$ a una temperatura de 20 ${ }^{\circ} \mathrm{C}$. Esta información es entregada por el fabricante sin ningún tipo de intervalo de confianza o información sobre su distribución. Por lo anterior, se asume una distribución triangular para poder estimar la incertidumbre típica:

$u(\mathrm{cal})=\frac{0,05 \mathrm{~mL}}{\sqrt{6}}=0,020 \mathrm{~mL}$

Es importante mencionar que el argumento de una distribución triangular se fundamenta en que los procesos productivos controlados, como lo es la fabricación de equipo volumétrico, el valor nominal es más probable que los extremos (Ellison y Williams, 2010). Otro argumento valido es que una tolerancia de $\pm 0,05 \mathrm{~mL}$ es una especificación que debería ser modelada como una distribución uniforme, ya que cualquier bureta dentro del ámbito especificado de $\pm 0,05 \mathrm{~mL}$ pudo ser considerada por el fabricante como aceptable.

Una manera prudente de establecer el tipo de incertidumbre típica, la distribución y sesgo asociado a la medición de volumen es calibrando el equipo volumétrico. Estos servicios de calibración son realizados por un Instituto 
Nacional de Metrología (INM) o por laboratorios secundarios de calibración que demuestren competencia en este tipo de calibración.

\section{Temperatura}

Los fabricantes de equipo volumétrico realizan la calibración del instrumento a una temperatura de $20{ }^{\circ} \mathrm{C}$. Sin embargo, al utilizar el equipo volumétrico en las operaciones cotidianas de laboratorio, usualmente se trabaja con variaciones de temperatura límites de $\pm 4{ }^{\circ} \mathrm{C}$. Por consiguiente, producto del coeficiente de expansión volumétrico del líquido $(\alpha)$ se genera una variación en el volumen medido $(\Delta V)$. De la definición física de $\alpha$ podemos establecer que:

$\alpha=\frac{1}{V} \cdot\left(\frac{\partial V}{\partial_{t}}\right)_{p} \approx \frac{1}{V} \cdot \frac{\Delta V}{\Delta T} \Rightarrow \Delta V=\alpha \cdot V \cdot \Delta T$

Por lo tanto, una manera simplificada para calcular la incertidumbre generada por este $\Delta V$ es de la siguiente manera:

$u(t e m)=\alpha \cdot V \cdot \frac{\Delta T}{2 \sqrt{3}}=2,1 \times 10^{-4} \mathrm{C}^{-1} \cdot 16,70 \mathrm{~mL} \cdot \frac{4^{\circ} \mathrm{C}}{2 \sqrt{3}}=0,0040 \mathrm{~mL}$

La expresión anterior asume que la disolución de $\mathrm{HCl}$ posee una concentración tan pequeña que puede utilizarse el coeficiente de expansión volumétrico del agua $\left(2,1 \times 10^{-4}{ }^{\circ} C^{-1}\right)$ para el cálculo de la incertidumbre, además se asume una distribución uniforme para la medición de la temperatura. El cálculo anterior es aplicable para el volumen consumido por el blanco, $V_{\text {blanco }}=0,20 \mathrm{~mL}$, obteniendo un valor de $u($ tem $)=0,000050 \mathrm{~mL}$.

La incertidumbre típica del volumen consumido, $u\left(V_{\text {consu }}\right)$, se puede calcular como:

$u\left(V_{\text {consu }}\right)=\sqrt{2 \cdot(0,020)^{2}+(0,004)^{2}+(0,000050)^{2}}=0,029 \mathrm{~mL}=2,9 \times 10^{-5} L$

\subsection{Concentración de la disolución de $\mathrm{HCl}$}

Para efectos de este ejemplo supondremos que la disolución de $\mathrm{HCl}$ proviene de una preparación previa, en donde la concentración de $\mathrm{HCl}$ fue determinada estandarizando la disolución con un patrón primario de $\mathrm{Na}_{2} \mathrm{CO}_{3}$. En las Figuras 2 y 3 se observan todas las fuentes de incertidumbre, para simplificar el ejercicio el valor final de la concentración de $\mathrm{HCl}$ y la incertidumbre típica que se empleará es:

$C_{H C l}=(0,10030 \pm 0,00024) \mathrm{mol} \mathrm{L}^{-1}$

\subsection{Masa molar del nitrógeno}

La incertidumbre de la masa molar del nitrógeno puede calcularse a partir de las incertidumbres de los pesos atómicos de la IUPAC (IUPAC, 1999). Un argumento válido para calcular la incertidumbre típica es asignarle a la incertidumbre del pesos atómico del nitrógeno una tendencia central siendo así una distribución triangular o normal. Asumiendo una distribución triangular, la incertidumbre típica se calcula como:

$u\left(M_{N}\right)=\frac{0,00007 \mathrm{~g} \mathrm{~mol}^{-1}}{\sqrt{ } 6}=2,9 \times 10^{-5} \mathrm{~g} \mathrm{~mol}^{-1}$

\subsection{Masa de muestra}

La medición de la masa de leche en polvo posee 3 fuentes de incertidumbre significativas, sin embargo el aporte de incertidumbre por repetibilidad de las determinaciones de masa pueden ser agrupado dentro de una sola componente de repetibilidad, como se puede apreciar en la Figura 3. Por lo tanto, las dos contribuciones de incertidumbre contempladas en esta magnitud de influencia son:

\section{Linealidad/calibración:}

Usualmente los fabricantes de balanzas establecen que la contribución por linealidad es de $\pm 0,15 \mathrm{mg}$, este valor consiste en la máxima diferencia entre la masa del patrón y la lectura que indica la balanza al medir este patrón. En este tipo de contribución es conveniente asumir una distribución uniforme, obteniendo una incertidumbre típica de: 
$u($ line $)=\frac{0,15 m g}{\sqrt{ } 3}=0,087 \mathrm{mg}$

Resolución:

Las balanzas analíticas empleadas en este tipo de ensayos químicos poseen una resolución o límite de escala de $0,1 \mathrm{mg}$ (cuyo semiámbito de resolución es la mitad de $0,1 \mathrm{mg}$ ). La incertidumbre típica para este tipo de contribución se puede estimar asumiendo una distribución uniforme obteniendo:

$u($ line $)=\frac{0,10 m g}{2 \sqrt{ } 3}=0,029 \mathrm{mg}$

La incertidumbre típica de la masa de muestra de leche, $u\left(m_{\text {muestra }}\right)$, se puede calcular como:

$u\left(m_{\text {muestra }}\right)=\sqrt{(0,087)^{2}+(0,029)^{2}}=0,091 \mathrm{mg}=$ $9,17 \times 10^{-5} g$

\subsection{Repetibilidad}

Los componentes de incertidumbre Tipo A, pueden ser agrupados como una sola fuente de incertidumbre por repetibilidad, como se puede observar en la Figura 4. A partir de los resultados obtenidos en la validación del método podemos establecer que el coeficiente de variación (C.V) del método es de 0,017 , por lo que la incertidumbre típica por repetibilidad se calcula como:

$u(r e p)=\frac{C \cdot V}{\sqrt{n}}=\frac{0,017}{\sqrt{3}}=0,010$

En el Cuadro 1 se muestra un resumen con los valores de la determinación de proteína en leche en polvo y las incertidumbres típicas de cada una de las magnitudes de influencia. Recuerde que el C.V es la relación de media aritmética de los resultados con la dispersión de estos (desviación típica) como se puede apreciar en la siguiente formula $C \cdot V=\bar{x}$

$$
\bar{s}
$$

\section{CÁLCULO DE LA INCERTIDUMBRE COMBINADA E INCERTIDUMBRE EXPANDIDA}

\subsection{Estimación de la incertidumbre combinada e incertidumbre expandida por el método GUM}

Los lineamientos de la GUM establecen que la combinación de las incertidumbres típicas se

Cuadro 1. Valores de la determinación de contenido de proteína en leche en polvo y sus incertidumbres.

\begin{tabular}{cccc}
\hline Símbolo & Descripción & Valor, $\boldsymbol{x}_{\boldsymbol{i}}$ & Incertidumbre típica, $\boldsymbol{u}\left(\boldsymbol{x}_{\boldsymbol{i}}\right)$ \\
\hline$V_{\text {consu }}$ & $\begin{array}{c}\text { volumen consumido de } \\
\text { la disolución de } \mathrm{HCl} \\
C_{H C l}\end{array}$ & $0,01647 \mathrm{~L}$ & $0,000029 \mathrm{~L}$ \\
$M_{N}$ & $\begin{array}{c}\text { concentración de la } \\
\text { disolución de } \mathrm{HCl} \\
\text { masa molar del } \\
\text { nitrógeno }\end{array}$ & $0,10030 \mathrm{~mol} \mathrm{~L}^{-1}$ & $0,00024 \mathrm{~mol} \mathrm{~L}^{-1}$ \\
rep & $\begin{array}{c}\text { factor de repetibilidad } \\
\text { masa de muestra de } \\
\text { leche }\end{array}$ & $14,00674 \mathrm{~g} \mathrm{~mol}^{-1}$ & $0,000029 \mathrm{~g} \mathrm{~mol}^{-1}$ \\
$M_{\text {muestra }}$ & factor de conversión de \\
$N$ a proteína & $1,0517 \mathrm{~g}$ & 0,010 \\
$F$ & 6,38 & $0,000092 \mathrm{~g}$ \\
\hline
\end{tabular}


puede realizar utilizando las mismas reglas empleadas para la propagación del error, combinando variaciones aleatorias debidas a varias causas. En la GUM el cálculo de la incertidumbre típica combinada se realiza de la siguiente manera:

$$
u_{c}(y)=\sqrt{\sum_{i=1}^{N}\left[\frac{\partial f}{\partial x_{i}}\right]^{2} \cdot u^{2}\left(x_{i}\right)}=\sqrt{\sum_{i=1}^{N} C_{i}^{2} \cdot u^{2}\left(x_{i}\right)}
$$

Donde $y$ es la estimación del mensurando $Y$ (ver Ecuación 2), $C_{i}=\partial f / \partial x_{i}$ es el coeficiente de sensibilidad; el cual describe cómo varía la estimación de salida "y" en función de la variación de los valores de las estimaciones de entrada $x_{1}$, $x_{2}, \ldots, x_{N}$. La expresión anterior es aplicable para aquellos casos en donde las magnitudes de entrada sean independientes, en otras palabras que no existe dependientes entre si o correlacionadas (ISO, 1995).

A partir de la Ecuación (3) podemos calcular los coeficientes de sensibilidad para cada una de las magnitudes de entrada de la determinación del contenido de proteína en leche en polvo:

$$
\begin{aligned}
& c_{v_{\text {consu }}}=\frac{\partial \% \text { Proteína }}{\partial V_{\text {consu }}}=\frac{C_{H C l} \cdot M_{N} \cdot F \cdot r e p \cdot 100}{m_{\text {muestra }}}=852 \mathrm{~L}^{-1} \\
& c_{C_{H C l}}=\frac{\partial \% \text { Proteína }}{\partial C_{H C l}}=\frac{V_{\text {consu }} \cdot M_{N} \cdot F \cdot r e p \cdot 100}{m_{\text {muestra }}}=140 \mathrm{Lmol}^{-1} \\
& c_{M_{N}}=\frac{\partial \% \text { Proteína }}{\partial M_{N}}=\frac{V_{\text {consu }} \cdot C_{H C l} \cdot F \cdot r e p \cdot 100}{m_{\text {muestra }}}=1 \mathrm{~mol} \mathrm{~g}^{-1} \\
& c_{m_{\text {muestra }}}=\frac{\partial \% \text { Proteína }}{\partial m_{\text {muestra }}}=\frac{V_{\text {consu }} \cdot M_{N} \cdot F \cdot \text { rep } \cdot 100}{m^{2}{ }_{\text {muestra }}}=13 \mathrm{~g}^{-1} \\
& c_{\text {rep }}=\frac{\partial \% \text { Proteína }}{\partial \text { rep }}=\frac{V_{\text {consu }} \cdot C_{H C l} \cdot M_{N} \cdot F \cdot \text { rep } \cdot 100}{m_{\text {muestra }}}=14
\end{aligned}
$$

Calculados los coeficientes de sensibilidad, se calcula la incertidumbre típica combinada, el cálculo se describe a continuación:
El paso final es multiplicar la incertidumbre típica combinada por un factor de cobertura $(\mathrm{k})$ para obtener la incertidumbre expandida. La incertidumbre expandida es necesaria para proveer un intervalo con el cual se espera abarcar una larga fracción de valores de distribución que pueden ser razonablemente atribuidos al mensurando. (ISO, 1995). Para efectos de este ejemplo, asumiremos un $\mathrm{k}=2$ correspondiente a una probabilidad de cobertura del $95 \%$ para una distribución normal, por lo que la incertidumbre expandida de la medición se calcula como:

$U=k \cdot u_{c}(y)=k \cdot u_{c}(\%$ Proteína $)= \pm 0,29 \mathrm{~g}^{100 \mathrm{~g}^{-1}}$

El Cuadro 2 muestra el presupuesto de incertidumbre y la estimación de la incertidumbre típica combinada para la determinación del contenido de proteína en leche en polvo, basándose en el método propuesto por la GUM.

\subsection{Estimación de la incertidumbre combinada e incertidumbre expandida según por el método de Kragten}

La estimación de la incertidumbre por aproximación numérica es un método rápido y fácil, posee la ventaja, en comparación con el método diferencial propuesto por la GUM, de necesitar únicamente los valores de las magnitudes de entrada y sus incertidumbres típicas.

Asumiendo que $y=f\left(x_{i}, x_{2}, \ldots, x_{N}\right)$ es lineal en $x_{i}$ o que la incertidumbre típica de cada magnitud de entrada, $u\left(x_{i}\right)$, es muy pequeña en comparación con el valor de la magnitud de entrada $\left(x_{i}\right)$, los coeficientes de sensibilidad pueden ser aproximados de la siguiente manera (Kragten, 1994):

$$
\frac{\partial f}{\partial x} \approx \frac{y\left(x_{i}+u\left(x_{i}\right)\right)-y\left(x_{i}\right)}{u\left(x_{i}\right)}
$$

$$
\begin{aligned}
& u_{c}(\% \text { Proteína }) \\
& =\sqrt{C_{V_{\text {consu }}}^{2} \cdot u^{2}\left(V_{\text {consu }}\right)+C_{H C l}^{2} \cdot u^{2}(H C l)+C_{M_{N}}^{2} \cdot u^{2}\left(M_{N}\right)+C_{m_{\text {muestra }}}^{2} \cdot u^{2}\left(m_{\text {muestra }}\right)+C_{\text {rep }}^{2} \cdot u^{2}(\text { rep })} \\
& u_{c}(\% \text { Proteína }) \\
& =\sqrt{6,11 \times 10^{-4}+1,13 \times 10^{-3}+8,45 \times 10^{-10}+1,42 \times 10^{-6}+1,97 \times 10^{-2}}=0,146 \mathrm{~g} 100 \mathrm{~g}^{-1}
\end{aligned}
$$


Cuadro 2. Presupuesto y estimación de la incertidumbre de la determinación del contenido de proteína en leche en polvo por el método AOAC 930.29

\begin{tabular}{cccccc}
\hline Símbolo & Valor, $\boldsymbol{x}_{\boldsymbol{i}}$ & $\begin{array}{c}\text { Incertidumbre } \\
\text { típica, } \boldsymbol{u}\left(\boldsymbol{x}_{\boldsymbol{i}}\right)\end{array}$ & $\begin{array}{c}\text { Incertidumbre } \\
\text { típica, } \boldsymbol{u}\left(\boldsymbol{x}_{\boldsymbol{i}}\right)\end{array}$ & $\begin{array}{c}\text { Contribuciones } \\
\boldsymbol{u}_{\boldsymbol{i}}^{2}(\boldsymbol{y})=\boldsymbol{c i}^{2} \cdot \boldsymbol{u}^{2}\left(\boldsymbol{x}_{\boldsymbol{i}}\right)\end{array}$ & $\begin{array}{c}\text { Contribución } \\
\text { relativa, \% }\end{array}$ \\
\hline$V_{\text {consu }}$ & $0,016470 \mathrm{~L}$ & $0,000029 \mathrm{~L}$ & 852 & $6,11 \times 10^{-4}$ & 2,85 \\
$C_{H C l}$ & $0,10030 \mathrm{~mol} \mathrm{~L}^{-1}$ & $0,00024 \mathrm{~mol} \mathrm{~L}^{-1}$ & 140 & $1,13 \times 10^{-3}$ & 5,27 \\
$M_{N}$ & $14,006740 \mathrm{~g}$ & $0,000029 \mathrm{~g} \mathrm{~mol}^{-1}$ & 1 & $8,45 \times 10^{-10}$ & 0,00 \\
$M_{\text {muestra }}$ & $1,0517 \mathrm{~g}$ & $0,000092 \mathrm{~g}$ & 13 & $1,42 \times 10^{-6}$ & 0,007 \\
rep & 1,000 & 0,010 & 14 & $1,97 \times 10^{-2}$ & 91,87 \\
& & $\boldsymbol{\Sigma}\left[\boldsymbol{u}_{\boldsymbol{i}}^{2}(\boldsymbol{y})\right]$ & $2,14 \times 10^{-2}$ & 0,146 \\
\hline
\end{tabular}

Fuente: Elaboración propia, 2012.

Sustituyendo la aproximación anterior en la definición de la GUM, Ecuación (5), obtenemos que:

$u_{c}(y)=\sqrt{\sum_{i=1}^{N}\left[\frac{\partial f}{\partial x_{i}}\right]^{2} \cdot u^{2}\left(x_{i}\right)}=\sqrt{\sum_{i=1}^{N}\left[\frac{y\left(x_{i}+u\left(x_{i}\right)\right)-y\left(x_{i}\right)}{u\left(x_{i}\right)}\right]^{2} \cdot u^{2}\left(x_{i}\right)}$

Simplificando los términos de la Ecuación (7) se obtiene la ecuación para calcular la incertidumbre típica combinada por el método de Kragten:

$u_{c}(y)=\sqrt{\sum_{i=1}^{N}\left[y\left(x_{i}+u\left(x_{i}\right)\right)-y\left(x_{i}\right)\right]^{2}}$

Debe quedar claro, que esta aproximación sólo es aplicable a modelos lineales o valores pequeños de $u\left(x_{i}\right) / x_{i}$. De la Ecuación (8) se puede establecer que la contribución de incertidumbre de una magnitud de entrada es:

$u_{i}^{2}(y)=\underbrace{c_{i}^{2} \cdot u^{2}\left(x_{i}\right)}_{\text {GUM }}=[\underbrace{y\left(x_{i}+u\left(x_{i}\right)\right)-y\left(x_{i}\right)}_{\text {Kragten }}]^{2}$

Donde $y\left(x_{i}\right)$ es la expresión matemática del mensurando evaluada en la variable $x_{i}, y\left(x_{i}+\right.$ $\left.u\left(x_{i}\right)\right)$ es la expresión matemática del mensurando evaluada en la variable $x_{i}+u\left(x_{i}\right)$.

Para mayor comprensión de lo discutido anteriormente, en el siguiente ejemplo se ilustra como calcular la contribución de incertidumbre generada por el volumen consumido de $\mathrm{HCl}\left(V_{\text {cons }}\right)$, utilizando la aproximación numérica de Kragten.

\subsection{Contribución de incertidumbre del Volumen consumido de $\mathbf{H C l}$}

La contribución de incertidumbre del volumen consumido de $\mathrm{HCl}$ se puede realizar mediante tres simples pasos que se describen a continuación.

\section{Mensurando $y\left(x_{\mathrm{i}}\right)$}

Se calcula el valor del mensurando empleando el modelo matemático descrito en la Ecuación (3) y evaluando las variables: $V_{\text {consu }}, C_{H C l}, M_{N}, m_{\text {muestra }}$, rep:

\%Proteína $\left(V_{\text {consu }}, C_{H C l}, M_{N}, m_{\text {muestra }}\right.$, rep $)=\frac{V_{\text {consu }} \cdot C_{H C l} \cdot M_{N} \cdot F \cdot \text { rep }}{m_{\text {muestra }}} \cdot 100$ $=\frac{0,016470 \cdot 0,10030 \cdot 14,006740 \cdot 6,38}{1,0517} \cdot 100=14,03655 \mathrm{~g} 100 \mathrm{~g}^{-1}$

Mensurando evaluado como $y\left(x_{i}+u\left(x_{i}\right)\right)$

Conociendo el volumen consumido de $\mathrm{HCl} V_{\text {cons }}=0,01647 \mathrm{~L}$ y que su incertidumbre es $u\left(V_{\text {cons }}\right)=0,000029 L$, se puede calcular el mensurando empleando el modelo matemático descrito en la Ecuación (3) y evaluando las variables $\left(V_{\text {consu }}+u\left(V_{\text {cons }}\right)\right), C_{H C l}, M_{N}, m_{\text {muestra }}$, rep, como se muestra a continuación: 
\%Proteína $\left(\left(V_{\text {consu }}+u\left(V_{\text {cons }}\right)\right) \cdot C_{H C l} \cdot M_{N} \cdot m_{\text {muestra }} \cdot r e p\right)$

$=\frac{\left[V_{\text {cons }}+u\left(V_{\text {cons }}\right)\right] \cdot C_{H C l} \cdot M_{N} \cdot F \cdot \text { rep }}{m_{\text {mistra }}} \cdot 100$

$=\frac{[0,016470+0,000029] \cdot 0,10030 \cdot 14,006740 \cdot 6,38}{1,0517} \cdot 100=14,06127 g_{100 g^{-1}}$

\section{Contribución de incertidumbre}

Empleando la Ecuación (9) y los resultados obtenidos anteriormente la contribución de incertidumbre para la magnitud de $V_{\text {cons }}$ se calcula de la siguiente forma:

$u_{i}^{2}(y)=\left[y\left(x_{i}+u\left(x_{i}\right)\right)-y\left(x_{i}\right)\right]^{2}=(14,03655-14,06127)^{2}$ $=6,11 \times 10^{-4}(g / 100 g)^{2}$

En el Cuadro 3 se muestra el cálculo de las contribuciones de incertidumbre de las otras magnitudes así como del presupuesto de incertidumbre y estimación de la incertidumbre de la determinación del contenido de proteína en leche.

Una forma práctica de poder realizar la estimación de la incertidumbre de la medición por el método de aproximación numérica, es construyendo una hoja de cálculo en MS Excel ®. A continuación se describe los pasos para construir la hoja de cálculo para la estimación de la incertidumbre del contenido de proteína en leche en polvo por el método de Kragten:

- Introduzca los valores de las magnitudes de entrada $\left(V_{\text {consu }}, C_{H C l}, M_{N}\right.$ rep, $\left.m_{\text {muestra }}\right)$ en la columna $B$ desde la celda $B 4$ hasta $B 8$, además introduzca estos en la segunda fila desde la celda $C 2$ hasta $G 2$. Los valores de las incertidumbres típicas de cada magnitud, $u\left(V_{\text {consu }}\right), u\left(C_{\mathrm{HCl}}\right), u\left(M_{N}\right), u($ rep $), u\left(m_{\text {muestra }}\right)$, introdúzcalos en la tercera fila desde la celda $C 3$ hasta G3. Copie los valores de la columna $B$ y colóquelos en las columnas $C$, $D, \ldots, G$, como se muestra en la Figura 5a. En la celda $B 9$ introduzca la fórmula para el cálculo del mensurando. Para la determinación de proteína la ecuación viene dada por

Cuadro 3. Presupuesto y estimación de la incertidumbre de la determinación del contenido de proteína en leche por el método AOAC 930.29 empleando el método de aproximación numérica de Kragten.

\begin{tabular}{|c|c|c|c|c|c|}
\hline Símbolo & Valor, $\mathbf{x}_{\mathbf{i}}$ & $\begin{array}{l}\text { Incertidumbre } \\
\text { típica, } \mathbf{u}\left(\mathbf{x}_{\mathbf{i}}\right)\end{array}$ & $\mathbf{y}\left(\mathbf{x}_{\mathbf{i}}\right)$ & $\mathbf{y}\left(\mathbf{x}_{\mathbf{i}}+\mathbf{u}\left(\mathbf{x}_{\mathbf{i}}\right)\right)$ & $\begin{array}{c}\text { Contribuciones } \\
u_{i}^{2}(y)=\left[y\left(x_{i}+u\left(x_{i}\right)-\right.\right. \\
\left.y\left(x_{i}\right)\right]^{2}\end{array}$ \\
\hline$V_{\text {consu }}$ & $0,016470 L$ & $0,000029 L$ & 14,03655 & 14,06127 & $6,11 \times 10^{-4}$ \\
\hline$C_{H C l}$ & $0,10030 \mathrm{~mol} \mathrm{~L}^{-1}$ & $0,00024 \mathrm{~mol} L^{-1}$ & 14,03655 & 14,03658 & $1,13 \times 10^{-3}$ \\
\hline$M_{N}$ & $14,006740 \mathrm{~g} \mathrm{~mol}^{-1}$ & $0,000029 \mathrm{~g} \mathrm{~mol}^{-1}$ & 14,03655 & 14,17692 & $8,45 \times 10^{-10}$ \\
\hline$m_{\text {muestra }}$ & $1,0517 g$ & $0,000052 \mathrm{~g}$ & 14,03655 & 14,03532 & $1,51 \times 10^{-6}$ \\
\hline \multirow[t]{4}{*}{ rep } & 1,000 & 0,010 & 14,03655 & 14,17692 & $1,97 \times 10^{-2}$ \\
\hline & & & & $\Sigma\left[u_{i}^{2}(y)\right]$ & $2,14 \times 10^{-2}$ \\
\hline & & & & $u_{c}(y)$ & 0,146 \\
\hline & & & & $\mathrm{U} ; \mathrm{k}=2$ & 0,29 \\
\hline
\end{tabular}


a)

\begin{tabular}{|c|c|c|c|c|c|c|}
\hline 4 & A & B & $\mathrm{C}$ & D & $E$ & $\mathrm{~F}$ \\
\hline 1 & Valor & 0,01647 & 0,1003 & 14,00674 & 1 & 1,0517 \\
\hline 2 & Incertidumbre & 0,000029 & 0,00024 & 0,000029 & 0,01 & 0,000092 \\
\hline 3 & 0,01647 & $=\mathrm{B} 1+\mathrm{B} 2$ & $=\mathrm{A} 3$ & $=\mathrm{A} 3$ & $=\mathrm{A} 3$ & $=\mathrm{A} 3$ \\
\hline 4 & 0,1003 & $=\mathrm{A} 4$ & $=\mathrm{C} 1+\mathrm{C} 2$ & $=\mathrm{A} 4$ & $=\mathrm{A} 4$ & $=\mathrm{A} 4$ \\
\hline 5 & 14,00674 & $=\mathrm{A} 5$ & $=\mathrm{A} 5$ & $=\mathrm{D} 1+\mathrm{D} 2$ & $=\mathrm{A} 5$ & $=\mathrm{A} 5$ \\
\hline 6 & 1 & $=\mathrm{A} 6$ & $=\mathrm{A} 6$ & $=\mathrm{A} 6$ & $=\mathrm{E} 1+\mathrm{E} 2$ & $=\mathrm{A} 6$ \\
\hline 7 & 1,0517 & $=\mathrm{A} 7$ & $=\mathrm{A} 7$ & $=\mathrm{A} 7$ & $=\mathrm{A} 7$ & $=\mathrm{F} 1+\mathrm{F} 2$ \\
\hline $8=$ & $=A 3^{*} A 4^{*} \mathrm{~A} 5^{*} \mathrm{~A} 6^{*} 6,38 * 100 / \mathrm{A} 7$ & $=B 3 * B A+B 5 * B 6 * 6,38 * 100 / B 7$ & $=\mathrm{C} 3 * \mathrm{C} 4 * \mathrm{C} 5 * \mathrm{C} 6 * 6,38 * 100 / \mathrm{C} 7$ & $\mathrm{D} 3{ }^{*} \mathrm{D} 4{ }^{*} \mathrm{D} 5{ }^{*} \mathrm{D} 6 * 6,38 * 100 / \mathrm{D} 7$ & $=E 3^{*} E 4^{*} E 5^{*} E 6^{*} 6,38 * 100 / E 7$ & $=F 3^{*} F 4^{*} F 5^{*} F 6^{*} 6,38^{*} 100 / F 7$ \\
\hline 9 & & $=\mathrm{B} 8-\$ \mathrm{~A} \$ \mathrm{~B}$ & $=\mathrm{C} 8-\$ \mathrm{~A} \$ \mathrm{~B}$ & $=\mathrm{D} 8-\$ \mathrm{~A} \$ \mathrm{~S}_{8}$ & $=\mathrm{E} 8-\$ \mathrm{~A} \$ \mathrm{8}$ & $=\mathrm{F} 8-\$ \mathrm{~A} \$ \mathrm{~B}$ \\
\hline 10 & $=\operatorname{SUMA}(\mathrm{B} 10: \mathrm{F} 10)$ & = POTENCIA(B9;2) & $=$ POTENCIA $(\mathrm{C} 9 ; 2)$ & = POTENCIA(D9;2) & = POTENCIA(E9;2) & $=$ POTENCIA(F9;2) \\
\hline 11 & $=\mathrm{RAIZ}(\mathrm{A} 10)$ & $=\mathrm{B} 10 / \$ \mathrm{~A} \$ 10$ & $=\mathrm{C} 10 / \$ \mathrm{~A} \$ 10$ & $=\mathrm{D} 10 / \$ \mathrm{~A} \$ 10$ & $=\mathrm{E} 10 / \$ \mathrm{~A} \$ 10$ & $=\mathrm{F} 10 / \$ \mathrm{~A} \$ 10$ \\
\hline 12 & 2 & $\mathrm{k}$ & & & & \\
\hline 13 & $=\mathrm{A} 12 * \mathrm{~A} 11$ & $\mathrm{u}=\mathrm{k}^{*} \mathrm{u}_{\mathrm{c}}(\mathrm{y})$ & & & & \\
\hline
\end{tabular}

b)

\begin{tabular}{|c|c|c|c|c|c|c|c|}
\hline 4 & A & B & C & D & $E$ & $\mathrm{~F}$ & G \\
\hline 1 & & & $\mathrm{~V}_{\text {consu }}$ & $\mathrm{C}_{\mathrm{HCl}}$ & $\mathrm{M}_{\mathrm{N}}$ & rep & $\mathrm{m}_{\text {muestra }}$ \\
\hline 2 & & Valor & 0,01647 & 0,1003 & 14,00674 & 1 & 1,0517 \\
\hline 3 & & Incertidumbre & 0,000029 & 0,00024 & 0,000029 & 0,01 & 0,000092 \\
\hline 4 & $V_{\text {consu }}$ & 0,01647 & 0,016499 & 0,01647 & 0,01647 & 0,01647 & 0,01647 \\
\hline 5 & $\mathrm{C}_{\mathrm{HCl}}$ & 0,1003 & 0,1003 & 0,10054 & 0,1003 & 0,1003 & 0,1003 \\
\hline 6 & $\mathrm{M}_{\mathrm{N}}$ & 14,00674 & 14,00674 & 14,00674 & 14,006769 & 14,00674 & 14,00674 \\
\hline 7 & rep & 1 & 1 & 1 & 1 & 1,01 & 1 \\
\hline 8 & $m_{\text {muestra }}$ & 1,0517 & 1,0517 & 1,0517 & 1,0517 & 1,0517 & 1,051792 \\
\hline 9 & \%Proteína & 14,03655 & 14,06126611 & 14,07013784 & 14,03657994 & 14,17691638 & 14,03532 \\
\hline 10 & $u(y, x i)$ & & 0,02472 & 0,033586961 & $2,90617 \mathrm{E}-05$ & 0,140365509 & $-0,001227774$ \\
\hline 11 & $u(y)^{2}, u\left(y, x_{i}\right)^{2}$ & 0,02144 & $6,11 \mathrm{E}-04$ & $1,13 \mathrm{E}-03$ & $8,45 \mathrm{E}-10$ & $1,97 \mathrm{E}-02$ & $1,51 \mathrm{E}-06$ \\
\hline 12 & $\mathrm{u}_{\mathrm{c}}(\%$ Proteína $)$ & 0,146 & $2,85 \%$ & $5,26 \%$ & $0,00 \%$ & $91,88 \%$ & $0,007 \%$ \\
\hline 13 & $\mathrm{k}$ & 2 & & & & & \\
\hline 14 & U(\%proteína) & 0,29 & & & & & \\
\hline
\end{tabular}

Figura 5. Hoja de cálculo en MS Excel ${ }^{\circledR}$ para la estimación de la incertidumbre del contenido de proteína en leche en polvo por el método de Kragten. (a) Detalla los algoritmos introducidos en la hoja de cálculo (b) Muestra los resultados numéricos de la estimación de la incertidumbre por el método de Kragten.

Fuente: Elaboración propia, 2012. 
(a)

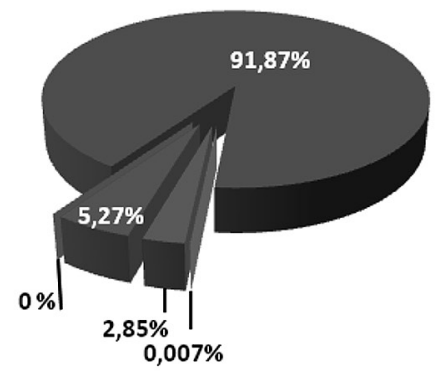

(b)

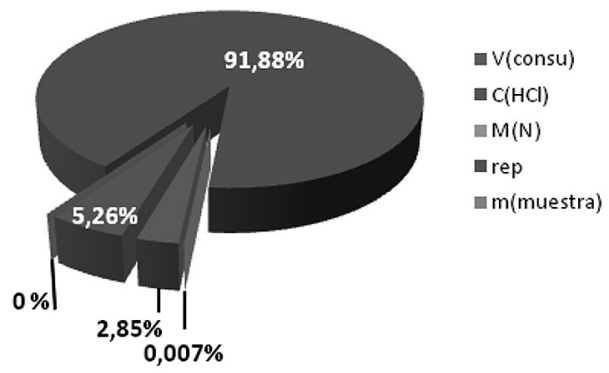

Figura 6.Contribuciones relativas de las principales fuentes de incertidumbre en la determinación del contenido de proteína en leche en polvo, (a) método GUM (b) método Kragten.

Fuente: Elaboración propia, 2012.

$=B 3 * B 4 * B 5 * B 6 * 6,38 * 100 / B 7$, copie la formula introducida en la celda $B 9$ e introdúzcala en las celdas C9, D9, E9, F9,G9.

- A la celda C4 sume el valor de la incertidumbre $\mathrm{u}\left(V_{\text {consu }}\right)$, a la celda D5 sume el valor de la incertidumbre de $\mathrm{u}(\mathrm{CHCl})$, siga realizando esta operación de manera escalonada hasta llegar a la celda G8 (ver Figura 5a).

- En la fila 10 realice la siguiente operación: sustraiga a la celda C9 el valor de la celda B9, realice esta sustracción desde la celda $\mathrm{C} 9$ hasta la celda G9 (por ejemplo la celda C9 se convierte en la siguiente fórmula de MS Excel,= C9-\$B\$9), ver Figura 5a.

- En la fila 11 calcule los residuales cuadráticos empleando la fórmula; =POTENCIA $(\mathrm{C} 10 ; 2)$. Realice la suma de los residuales cuadráticos en la celda B11, =SUMA (C11:G11), este cálculo se puede apreciar en la Figura 5a. La incertidumbre típica combinada se obtiene al aplicar la raíz cuadrada del resultado obtenido en la celda B11, =RAIZ (B11), coloque el resultado de esta operación en la celda A11.

- Por último, se puede calcular la incertidumbre expandida multiplicando la incertidumbre típica combinada contenida en la celda A11 por un factor de cobertura adecuado. Para efectos de este ejemplo se utilizará un $\mathrm{k}=2$ para un nivel de confianza del 95\%. La contribución relativa de cada fuente de incertidumbre $\left(u\left(x_{i}\right) / x_{i}\right)$ pueden ser calculadas relacionando los resultados obtenidos en la fila 11 con lo obtenido en la celda B11, por ejemplo para la contribución relativa para el volumen consumido la operación en la hoja de cálculo sería, $=\mathrm{C} 11 / \mathrm{B} \$ 11$, este resultado provee una forma sencilla de observar cuales componentes de incertidumbre son significativos.

Como se puede observar en la Figura 5b, el método de Kragten produce el mismo resultado (incertidumbre típica combinada e incertidumbre expandida) que el obtenido empleando el método diferencial de la GUM. En la Figura 6 se puede apreciar claramente este resultado, en el cual ambos métodos conducen a las mismas contribuciones relativas de incertidumbre.

\section{CONCLUSIONES}

La estimación de incertidumbre es un proceso en el que se debe tener claridad y comprensión del sistema de medición. Un enfoque analítico del sistema de medición entregará al analista fundamentos técnicos para establecer las fuentes de incertidumbre involucradas en el ensayo de análisis químico. Existen varios métodos para la estimación de la incertidumbre los cuales deben ser consultados a la hora de estimar la incertidumbre de medida. Las hojas de cálculo elaboradas con el método de Kragten pueden ser utilizadas como una herramienta simple y práctica para la estimación de la incertidumbre de medición, este 
método simplifica el cálculo de la incertidumbre típica combinada y disminuye posibles errores de cálculo como es habitual cometer en el método diferencial de la GUM. Se observó que el método de aproximación numérica genera estimaciones de incertidumbre prácticamente iguales a las obtenidas por el método diferencial propuesto por la GUM, alcanzando en términos relativos un error de $0,001 \%$ al comparar los resultados del método de Kragten con los obtenidos mediante el método diferencial propuesto por la GUM.

\section{REFERENCIAS BIBLIOGRÁFICAS}

Ellison, S.L.R, Williams, A. (2010). Quantifying Uncertainty in Analytical Measurement. Eurachem/CITAC.

Ellison, S. L. R. y Barwick, V. J. (1998). Estimating measurement uncertainty: reconciliation using a cause and effect approach. Accreditation Quality Assurance, 3 (3), 101 - 105.

ISO Guide 98. (1995).Guide to the Expression of Uncertainty in Measurement. Switzerland: International Organization for Standardization.

ISO Guide 99. (2007). International Vocabulary of Metrology - Basic and General
Concepts and Associated Terms (VIM). Switzerland: International Organization for Standardization.

International Union of Pure and Applied Chemistry. (1999). Commission on Atomic Weights and Isotopic Abundances. Pure Appl. Chem. 71(8), 1593 - 1607.

Kragten, J.(1994). Calculating standard deviations and confidence intervals with a universally applicable spreadsheet technique. Analyst, 119 (10), $2161-2166$.

Association of Official Analytical Chemists. (1990). Protein in Dried Milk. 930.29.A. In: Official Methods of Analysis. Volume II: Food Composition and Additives; Natural Contaminants. Virginia: AOAC.

\section{SOBRE EL AUTOR}

\section{Bryan Calderón Jiménez,}

Licenciado en Química Industrial, investigador del departamento de Metrología en Química del Laboratorio Costarricense de Metrología, Ministerio de Economía, Industria y Comercio.

Teléfono: (506) 2283-6580, ext. 116

Correo electrónico: bcalderon@lacomet.go.cr 
